\title{
Effect of Lesions of the Ventrolateral Preoptic Nucleus on NREM and REM Sleep
}

\author{
Jun Lu, ${ }^{1}$ Mary Ann Greco, ${ }^{2}$ Priyattam Shiromani, ${ }^{2}$ and Clifford B. Saper ${ }^{1,3}$ \\ ${ }^{1}$ Department of Neurology and ${ }^{3}$ Program in Neuroscience, Beth Israel Deaconess Medical Center, Harvard Medical \\ School, Boston, Massachusetts 02215, and 2Department of Psychiatry, Brockton Veterans Administration Hospital, \\ Harvard Medical School, Boston, Massachusetts 02115
}

Neurons in the ventrolateral preoptic nucleus (VLPO) in rats show c-fos activation after sleep and provide GABAergic innervation of the major monoamine arousal systems, suggesting that they may be a necessary part of the brain circuitry that produces sleep. We examined the effects on sleep behavior in rats of cell-specific damage to the VLPO by microinjection of ibotenic acid. Severe lesions of the central cell cluster of the VLPO ( $~ 80-90 \%$ cell loss bilaterally) caused a $60-70 \%$ decrease in delta power and a 50-60\% decrease in nonrapid-eyemovement (NREM) sleep time $(p<0.001)$. The number of remaining Fos-immunoreactive neurons in the VLPO cell cluster was linearly related to NREM sleep time $(r=0.77 ; p<0.001)$ and total electroencephalogram delta power $(r=0.79 ; p<$ $0.001)$ but not to rapid-eye-movement (REM) sleep $(r=0.35$; $p>0.10)$. Lesions in the region containing scattered VLPO

The involvement of the preoptic area and adjacent basal forebrain in sleep regulation has been recognized since von Economo (1930) reported that lesions of this region cause prolonged insomnia in humans. Nauta (1946) demonstrated that preopticbasal forebrain lesions cause insomnia in rats, and Sterman and Clemente (1962a,b) demonstrated similar effects in cats. These experimental studies used electrolytic or mechanical lesions that not only destroyed neuronal cell bodies but also damaged fibers of passage. More recent studies using chemical toxins to ablate the neuronal cell bodies in this area in rats and cats have confirmed the production of insomnia (Szymusiak and McGinty, 1986; Sallanon et al., 1989; John and Kumar, 1998). However, these studies used large lesions and hence could not identify a specific neuronal population that was responsible for the deficit in sleep regulation.

Recently, Sherin et al. (1996) identified a specific population of neurons in the ventrolateral preoptic nucleus (VLPO) that show Fos immunoreactivity after sleep (sleep-positive neurons). The number of Fos-immunoreactive (-IR) neurons in this region was directly proportional to the number of minutes of sleep during the previous hour (Sherin et al., 1996), in good agreement with electrophysiological studies showing that sleep-active neurons, with firing rates two to three times faster during sleep than during

\footnotetext{
Received July 27, 1999; revised Feb. 8, 2000; accepted Feb. 17, 2000.

This work was supported by United States Public Health Service Grants HL60292 and MH55772. We thank Quan Hue Ha and Mihn Tuan Ha for excellent technical work.

Correspondence should be addressed to Dr. C. B. Saper, Department of Neurology, Beth Israel Deaconess Medical Center, 330 Brookline Avenue, Boston, MA 02215. E-mail: csaper@caregroup.harvard.edu.

Copyright (C) 2000 Society for Neuroscience $\quad 0270-6474 / 00 / 203830-13 \$ 15.00 / 0$
}

neurons medial or dorsal to the cell cluster caused smaller changes in NREM sleep time (24.5 or $15 \%$, respectively) but were more closely associated with loss of REM sleep $(r=0.74$; $p<0.01$ ). The insomnia caused by bilateral VLPO lesions persisted for at least 3 weeks. Lesions of the VLPO caused no change in mean body temperature or its circadian variation; after small lesions of the ventromedial preoptic nucleus, body temperature showed normal circadian variation but a wider temperature range, and sleep behavior was not affected. These experiments delineate distinct preoptic sites with primary effects on the regulation of NREM sleep, REM sleep, and body temperature.

Key words: preoptic area; hypothalamus; REM sleep; NREM sleep; thermoregulation; circadian rhythms

wakefulness, are particularly numerous in the VLPO (Alam et al., 1995; Szymusiak et al., 1998).

The sleep-positive neurons in the VLPO innervate the histaminergic neurons in the tuberomammillary nucleus (TMN) (Sherin et al., 1996). These projections originate from a dense cluster of VLPO neurons just lateral to the optic chiasm, as well as from a more diffuse population of cells that extends dorsally and medially from this cluster (the extended VLPO). Approximately $80 \%$ of the neurons in this population contain the inhibitory neurotransmitters GABA and galanin. Histaminergic neurons in the TMN are believed to play a key role in promoting wakefulness (Lin et al., 1988, 1996; Steininger et al., 1999); inhibition of these neurons by the VLPO is thought to play a major role in causing sleep. Steininger et al. (1997) and Yang and Hatton (1997) have shown that electrical stimulation in the VLPO region causes $\mathrm{GABA}_{\mathrm{A}}$ receptor-mediated hyperpolarization and inhibition of TMN neurons in brain slices.

These results suggest that the VLPO may play an important role in promoting sleep. The preoptic area, however, is also involved in thermoregulation, and alterations in body temperature can affect sleep (Szymusiak et al., 1991; McGinty et al., 1994). We therefore sought to characterize more precisely whether damage specifically to VLPO neurons may cause insomnia and whether this was associated with changes in body temperature. We placed small ibotenic acid lesions in the VLPO region and recorded both sleep and body temperature continuously for periods of 1-3 weeks. We further used Fos immunocytochemistry to correlate these responses with the number of remaining sleep-positive neurons in the VLPO central cluster and the extended VLPO. This histological marker allowed us to 
correlate precisely the anatomical location of the lesions with their effects on sleep.

\section{MATERIALS AND METHODS}

\section{Animals, surgery, and recording methods}

\section{Animals}

Pathogen-free adult male Sprague Dawley rats $(250-275 \mathrm{gm} ; n=82)$ purchased from Taconic (Germantown, NY) were housed individually and given access to food and water ad libitum. All animals were housed under controlled conditions ( $12 \mathrm{hr}$ of light starting at 07:00) in an isolated ventilated chamber maintained at $20-22^{\circ} \mathrm{C}$. All protocols were approved by the Institutional Animal Care and Use Committees of Beth Israel Deaconess Medical Center and Harvard Medical School.

\section{Electroencephalogram/electromyogram implantation, sleep recording, and scoring}

The rats were anesthetized by intraperitoneal chloral hydrate (7\% in saline; $350 \mathrm{mg} / \mathrm{kg}$ ), prepared for aseptic surgery, and secured in a stereotaxic frame. Four electroencephalogram (EEG) electrodes were screwed onto the skull (two on the left and two on the right), two electromyogram (EMG) electrodes were placed under the nuchal muscles, and all leads were connected to a pedestal socket. Dental cement was then used to affix the EEG/EMG electrodes to the skull. A small area $(0.50 \mathrm{~cm})$ around the bregma was left without dental cement and filled with bone wax that was removable.

After a 6 d recovery period, animals were adapted to the EEG/EMG recording apparatus for $24 \mathrm{hr}$ before recordings began. The EEG/EMG signals were amplified by a polygraph (Grass) and digitized by an ICELUS (G System). Wake-sleep states were manually scored, by an observer who was not aware of the histological results of the animals, in $12 \mathrm{sec}$ epochs based on the digitized EEG/EMG of each rat. Wakefulness was identified by the presence of desynchronized-EEG and highEMG activity. Nonrapid-eye-movement (NREM) sleep was identified by the presence of a high-amplitude, slow-wave EEG and low-EMG activity relative to that of waking. Rapid-eye-movement (REM) sleep was identified by the presence of regular theta activity on EEG, coupled with low-EMG activity relative to that of NREM sleep. When two states (for example, NREM sleep and wake) occurred within a $12 \mathrm{sec}$ epoch, the epoch was scored for the state that predominated.

\section{Ibotenic acid injection}

Animals were anesthetized with chloral hydrate, bone wax was removed, the skull was reexposed, and a fine glass micropipette $(10-20 \mu \mathrm{m}$ tip diameter) was lowered into the VLPO area on each side of the brain stereotaxically. The coordinates used for the VLPO (Paxinos and Watson, 1986) were anteroposterior, $-0.6 \mathrm{~mm}$; dorsoventral, $-8.5 \mathrm{~mm}$; and mediolateral, $\pm 1.0 \mathrm{~mm}$ with reference to the bregma, with the tooth bar at $-3.3 \mathrm{~mm}$. The coordinates for medial preoptic control injections were anteroposterior, $-0.4 \mathrm{~mm}$; dorsoventral, $-8.0 \mathrm{~mm}$; and mediolateral, $\pm 0.4 \mathrm{~mm}$; and the coordinates for ventromedial preoptic injections were anteroposterior, $-0.2 \mathrm{~mm}$; dorsoventral, $-8.2 \mathrm{~mm}$; and mediolateral, $\pm 0.2 \mathrm{~mm}$. Fifteen nanoliters of ibotenic acid solution $(10 \mathrm{nmol}$; Sigma) were injected by air pressure through the pipette. The actual injection volume was measured by displacement of the solution meniscus within a micropipette of known internal diameter, as visualized with an operating microscope fitted with an eyepiece micrometer (see also Scammell et al., 1998). After 2 min, the pipette was slowly withdrawn. The wound was treated with teramycin ointment, filled with sterile bone wax (Ethicon), and closed with wound clips.

\section{Perfusion, tissue sectioning, and staining}

\section{Perfusion and tissue sectioning}

To identify sleep-positive neurons in the VLPO, we deeply anesthetized the rats with chloral hydrate between 10:00 and 12:00 noon when they had been behaviorally asleep for most of the previous hour and perfused the animals with saline $(100 \mathrm{ml})$ followed by $10 \%$ formalin $(300 \mathrm{ml})$ transcardially. The brains were cut in the coronal plane on a freezing microtome into four evenly spaced series of $40 \mu \mathrm{m}$ sections.

\section{Immunohistochemistry}

Sections were washed in PBS (three changes) and incubated in PBS containing $0.3 \%$ Triton X-100 and goat serum (PGT) for $1 \mathrm{hr}$ at room temperature, followed by the primary antiserum (Fos, Ab-5 rabbit poly- clonal serum; 1:150,000; Oncogene) in the same diluent for $2 \mathrm{~d}$ at room temperature. Sections were then washed in PBS and incubated in biotinylated secondary goat anti-rabbit IgG antiserum (1:1000) in PGT for $1 \mathrm{hr}$ and washed in PBS and incubated in ABC reagents (1:500; Vector Laboratories, Burlingame, CA) for $1 \mathrm{hr}$. Sections were then washed again and incubated in a solution of $0.06 \%$ 3,3-diaminobenzidine tetrahydrochloride (DAB; Sigma), $0.005 \%$ cobalt chloride, and $0.001 \%$ nickel ammonium sulfate in PBS plus $0.012 \% \mathrm{H}_{2} \mathrm{O}_{2}$ for $10 \mathrm{~min}$. Finally, the sections were mounted on glass slides, dehydrated in graded alcohols, cleared in xylene (10 min; twice), and coverslipped.

\section{Nissl staining}

An alternate series of sections adjacent to those that were Fos-stained was mounted on gelatin-coated slides, washed in $\mathrm{H}_{2} \mathrm{O}$, and washed in PBS. Sections were then incubated in $0.25 \%$ thionin in $0.1 \mathrm{~m}$ acetate buffer solution for $2 \mathrm{~min}$, differentiated in graded ethanols, and cleared in xylene before being coverslipped.

\section{Recording of sleep and temperature physiology}

Experiment 1: measurement of short-term effects $(4-6 d)$ of bilateral VLPO lesions on sleep

The prelesion control EEG/EMG of the rats was continuously recorded for $24 \mathrm{hr}(13: 00-13: 00) 6 \mathrm{~d}$ after the EEG/EMG implantation. The rats then received bilateral injections of ibotenic acid targeted at the VLPO $(n=48)$ or the medial preoptic area $(n=8)$. Four to $6 \mathrm{~d}$ later, the $\mathrm{EEM} / \mathrm{EMG}$ was continuously recorded again for $24 \mathrm{hr}$ (13:00-13:00). Rats were then perfused on day 7 after lesion placement, as described above.

\section{Experiment 2: measurement of long-term effects (1-3 weeks)} of bilateral VLPO lesions on sleep and body temperature

The preoptic area, including the ventromedial preoptic region just medial to the VLPO, contains thermosensitive neurons that are responsible for temperature regulation. To determine whether lesions of thermoregulatory neurons medial to the VLPO might contribute to sleep alterations or conversely whether VLPO lesions affected sleep behavior by altering thermoregulation, 26 additional rats were implanted for EEG/EMG recording as well as receiving intraperitoneal temperature telemetry transmitters (VM-FH; Mini-Mitter, Sunriver, OR). Injections of $15 \mathrm{nl}$ of ibotenic acid $(10 \mathrm{nmol})$ were made into the VLPO region in 15 animals at the time of the initial surgery. As controls, 5 animals received injections of ibotenic acid into the ventromedial preoptic region, and 6 rats received $15 \mathrm{nl}$ injections of saline into the VLPO.

To determine whether the changes in sleep behavior and regulation of body temperature were long-lasting, EEG/EMG and body temperature were recorded for $24 \mathrm{hr}$ (from 13:00 to 13:00) at the end of the first, second, and third weeks after VLPO lesions. The rats were later perfused between 10:00 and 12:00 noon as described above, and their brain sections were examined for Fos and Nissl staining.

\section{Determination of involvement of VLPO neurons by lesions}

All cell counts were done before analyzing the physiological data so that observers were blinded to the behavioral responses.

\section{Fos-IR neurons}

Fos-IR neurons in the VLPO cell cluster were quantified as described in Sherin et al. (1996). We counted all neurons with deeply black-stained nuclei that were within a counting box containing the VLPO cluster, an area that was framed by the lateral edge of the optic chiasm and extended $300 \mu \mathrm{m}$ dorsally and $500 \mu \mathrm{m}$ laterally in three coronal sections (spaced $160 \mu \mathrm{m}$ apart). In our previous studies (Sherin et al., 1996, 1998), we found that the numbers of Fos-IR neurons in this box are linearly related to sleep behavior. Most of the Fos-IR neurons within this box in sleeping animals were located within the pyramidal-shaped VLPO cluster. However, we found that smaller numbers of Fos-IR neurons after sleep were also located in the region dorsal and medial to the counting box and VLPO cluster. Because some neurons in this region contain galanin and glutamic acid decarboxylase immunoreactivity and project to the histaminergic tuberomammillary nucleus, we had hypothesized previously that they belong to the VLPO nucleus but represent a dorsal and a medial extension (Sherin et al., 1998). To test quantitatively the relationship of this Fos expression and sleep responses in our animals, we constructed a box $300 \mu \mathrm{m}$ in dorsoventral height that extended $400 \mu \mathrm{m}$ 
medially from the VLPO counting box to quantify the neurons in the medial-extended VLPO. We similarly constructed a counting box just dorsal to the first two, centered on the border between the VLPO and medial-extended VLPO and $200 \mu \mathrm{m}$ in width and $300 \mu \mathrm{m}$ in height, to delineate the dorsal-extended VLPO. We used the same sections in which we counted cells in the VLPO cluster to count the cells in the dorsal- and medial-extended VLPO.

We measured the size of nuclei in VLPO neurons in control rats, in animals with partial lesions ( $<80 \%$ bilateral), and in animals with $80-$ $90 \%$ lesions bilaterally. We found no differences of the sizes of Fospositive nuclei between these groups. Thus, it was not necessary to use a correction formula to compare cell counts between groups. Because the Fos antibodies do not penetrate tissue evenly, we did not use stereological counting methods.

\section{Nissl staining}

The VLPO cluster is recognizable in normal brains as a dense cellular aggregation whose neurons are slightly larger and more darkly stained than those in the adjacent preoptic region but smaller and less darkly stained than those in supraoptic neurons. However, in lesioned animals these distinctions can be difficult. Furthermore, the VLPO is surrounded by other Nissl-stained cell groups, so that it is impractical to use a large rectangular counting box, as we did in the Fos studies to define the VLPO. We therefore counted all Nissl-stained neuronal nuclei within an isosceles triangular area with its base $200 \mu \mathrm{m}$ in width running along the base of the brain starting at the lateral edge of the supraoptic nucleus and its apex $250 \mu \mathrm{m}$ dorsal to the center of the base. This triangle was very close in size, shape, and location to the VLPO cluster, and this procedure avoided making subjective counts of which neurons were within the VLPO after the lesions. Interestingly, neurons within the VLPO triangle tended to be resistant to ibotenic acid, so that this structure often stood out as intact when neurons in the regions surrounding the VLPO were destroyed. This property was very useful in allowing us to isolate the quantitative relationship between the loss of VLPO cluster neurons and sleep behavior. However, neurons of the dorsal- and medial-extended VLPO were too diffuse to be recognized in Nissl-stained sections. Because neuronal nuclei in the VLPO cluster were not different in size after lesions, we did not use a correction factor to compare the different groups. We could not use stereological methods because the lesion damage made it difficult to define the reference space accurately.

\section{Determination of the lesion area}

We used the area of neuronal loss and gliosis demonstrated on Nissl staining to determine the lesion region. Lesions were plotted using a camera lucida device (Fig. 1).

\section{Analysis of the relationship between loss of the VLPO cluster and extended VLPO neurons and effects on sleep and body temperature}

To assess the short-term effects of VLPO lesions on sleep, we used a total of 56 rats. In eight cases, the lesion was aimed at the medial preoptic nucleus as controls. The remaining 48 animals, in which the VLPO was targeted, were divided into three groups, based on examination of the histology. In group I, the VLPO cluster was intact ( $<50 \%$ cell loss on each side of the brain, as assessed in Nissl-stained sections; $n=9$ ); in group II, a unilateral lesion of the VLPO cluster was present $(>50 \%$ cell loss on one side; $n=10$ ); and in group III, bilateral lesions of the VLPO cluster were documented ( $>50 \%$ cell loss bilaterally; $n=29)$. We further subdivided group III into a set of seven rats with nearly complete bilateral lesions of the VLPO cluster ( $>80 \%$ cell loss on each side). For a variety of technical reasons, eight animals could not be evaluated adequately and were dropped from further analysis.

\section{Effect of VLPO lesions on sleep architecture}

We plotted the percentage of NREM sleep per hour and the percentage of REM sleep per hour for $24 \mathrm{hr}$, both before and 4-6 d after lesion placement, for the seven animals in group III with $>80 \%$ cell loss in the VLPO cluster bilaterally. We used an ANOVA and the nonparametric Mann-Whitney test to determine significant differences in the paired data.

\section{The relationship between Fos-IR neurons in the VLPO and sleep}

To determine the effect of VLPO lesions specifically on sleep behavior, we correlated numbers of Fos-IR neurons in the VLPO cluster and the extended VLPO with EEG delta power, NREM sleep time, and REM sleep time. Our preliminary experiments showed that unilateral VLPO lesions did not affect total sleep time. Thus, we only used data from rats with bilateral lesions ( $>50 \%$ neuron loss bilaterally). The number of Fos-IR neurons and Nissl-stained neurons are represented as the mean number of cells per section (on one side of the brain).

The relationship of the number of sleep-positive neurons in the VLPO cluster and extended VLPO and delta power in the $24 \mathrm{hr} E E G$. Delta power $(0.5-4.0 \mathrm{~Hz})$ occurs mainly during stage 3-4 NREM sleep; thus, the amount of delta power in the EEG can be used as a measure of the amount of deep sleep. We used the ICELUS program to calculate the delta power summed over $24 \mathrm{hr}$ and to compare the change between the baseline EEG versus the EEG $6 \mathrm{~d}$ after lesion placement. The delta power change was then plotted against the number of sleep-positive (Fos-IR) neurons in the VLPO cluster or extended VLPO of each animal, and the correlation coefficient across the group was determined.

The relationship between the number of sleep-positive neurons in the $V L P O$ cluster and extended VLPO and NREM sleep time. We plotted the number of Fos-IR neurons in the VLPO cluster and the extended VLPO against the percentage change in the duration of NREM sleep and calculated the correlation coefficient.

The relationship between the number of Fos-IR neurons in the VLPO cluster and extended VLPO and REM sleep time. We compared the number of Fos-IR cells in the VLPO cluster and the extended VLPO against the percentage reduction of REM sleep in all animals by calculating the correlation coefficient.

To determine the long-term effects of VLPO lesions, we used a total of 26 animals. We divided these into a group of 15 animals with $>50 \%$ bilateral lesions of the VLPO cluster, a group of 6 animals with $>50 \%$ bilateral ventromedial preoptic lesions, and 5 control rats with saline injections. The group with VLPO lesions was further subdivided into 4 rats with severe $(70-90 \%)$ neuron loss bilaterally in the VLPO cluster and 11 with less severe lesions. To determine sleep alteration, we compared NREM sleep of the rats with severe bilateral VLPO cluster lesions with that of control rats that had received sham (saline) injection. For linear regression analysis we plotted NREM sleep time against the loss of neurons in the VLPO cluster for the full group of rats with VLPO lesions of different degrees on both sides of the brain $(n=15)$ as well as for the rats with control injections $(n=5)$. Because we did not measure prelesion EEG in this group, delta power (which varies substantially between animals in part as a function of electrode placement and therefore can only be examined in paired data from the same animal) could not be used for comparison.

To determine alterations of body temperature, the mean body temperature across $24 \mathrm{hr}$ and the range of circadian variation in body temperature were calculated for each animal, and the mean for the controls was compared with that of the four animals with the most severe lesions of the VLPO cluster and the five animals with ventromedial preoptic lesions by using a $t$ test. In addition, the mean body temperature summated for each hour of the day was calculated for each group, and comparisons were made across groups by an ANOVA followed by $t$ tests. Finally, the variance of the body temperature was calculated for each animal, and the mean in each experimental group was calculated and compared with the controls by a $t$ test.

\section{The relationship between the number of Fos-IR neurons and the number of neurons revealed by Nissl staining in the VLPO cluster}

To determine whether the reduction in the number of Fos-IR neurons in the VLPO cluster after sleep accurately reflected cell loss in this structure, we calculated the correlation coefficient between these two measures.

\section{RESULTS}

\section{Experiment 1: short-term effects of bilateral lesions of the VLPO cluster and extended VLPO on sleep}

We attempted to destroy VLPO neurons while minimizing the damage to adjacent areas by using injection of $10 \mathrm{nmol}$ of ibotenic acid into the VLPO region (Figs. 1, 2C). Although the lesions that resulted were small (diameter, $\sim 400-800 \mu \mathrm{m}$ ), all extended beyond the VLPO cluster to involve adjacent areas to varying degrees. Furthermore, in most cases with lesions successfully 


\section{A $\quad 80-90 \%$ cell loss in the VLPO}
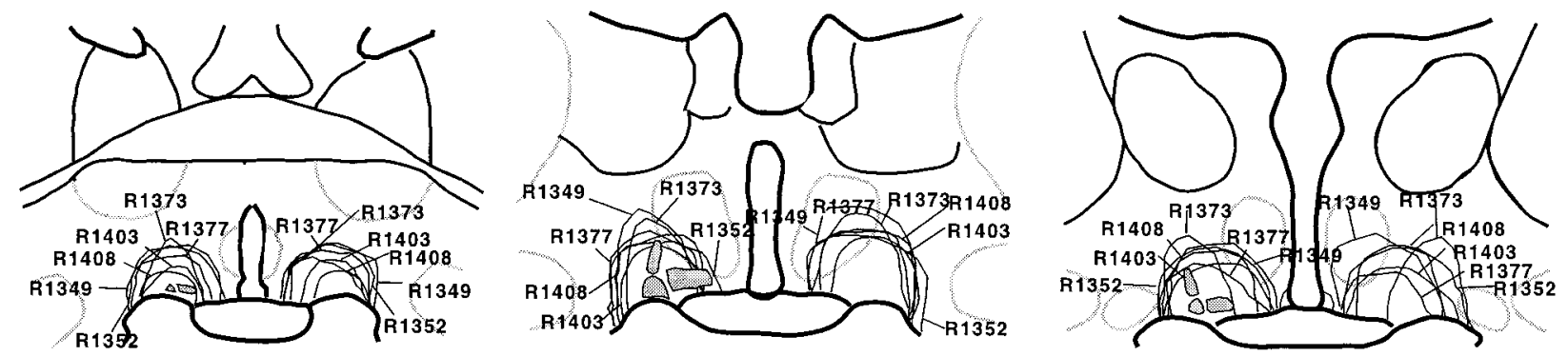

B $\quad 50-80 \%$ cell loss in the VLPO
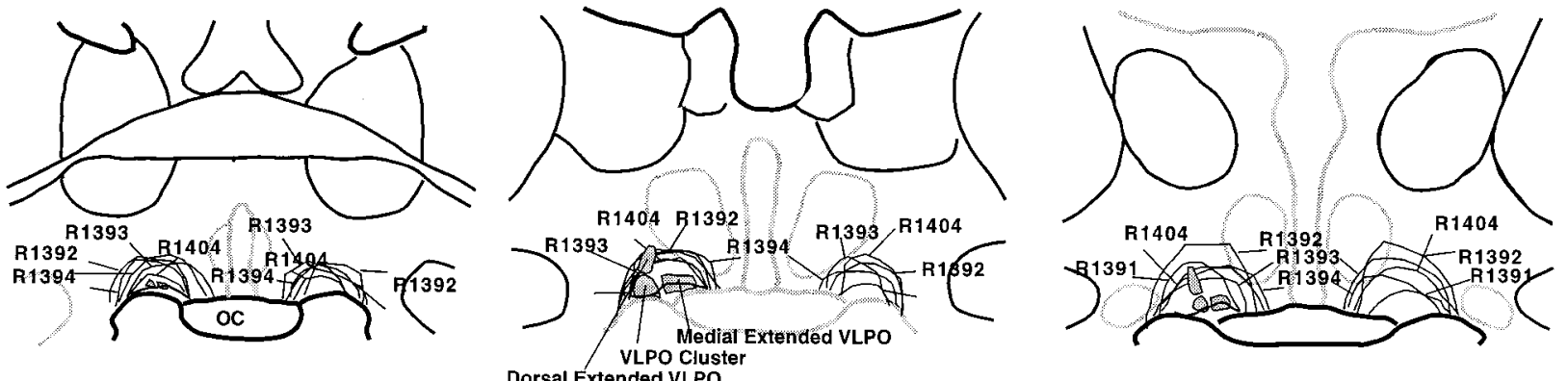

Dorsal Extended VLPO
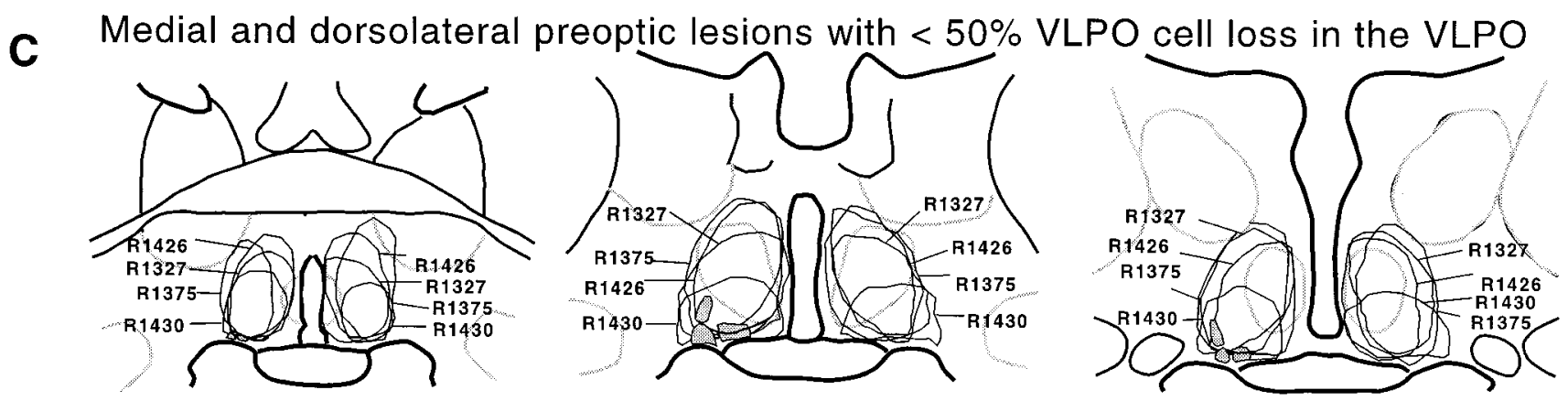

Figure 1. Camera lucida drawings illustrating the typical lesions of the VLPO cluster of $80-90 \%(A)$ and $50-80 \%(B)$ and control lesions in the medial preoptic area and dorsolateral preoptic area with $<50 \%$ cell loss in the VLPO cell cluster $(C)$, as assessed in Nissl-stained sections. $A$, The lesion areas in the animals that had $80-90 \%$ loss of the cells in the VLPO cluster ranged from 400 to $800 \mu \mathrm{m}$ in diameter and included the entire extent of the VLPO. $B$, Because the cells in the VLPO cluster were relatively resistant to ibotenic acid compared with the cells in the surrounding regions, even when the ibotenic acid injection was placed close to the base of the hypothalamus, substantial numbers of cells in the VLPO cluster often survived (50-80\% cell loss). $C$, Similarly when the lesion sites were placed just dorsal to the VLPO cluster, the areas around the VLPO were often devastated, but the majority of cells in the VLPO cluster survived. OC, Optic chiasm.

centered in the VLPO cluster, $\sim 10-20 \%$ of neurons in the VLPO cluster were still intact as determined by Nissl staining (Fig. 2C) and c-fos immunostaining (Fig. 2D). Hence, we used statistical analysis to compare the extent of injury to the VLPO cluster and extended VLPO with changes in sleep behavior. To control for damage extending beyond the VLPO cluster, we examined the sleep behavior of rats in which the lesions were mainly placed dorsal or medial to the VLPO cluster, involving the dorsal- or medial-extended VLPO, but with only unilateral or $<50 \%$ bilateral cell loss in the VLPO cluster (Fig. $2 F-I$ ). As expected, there was a strong linear correlation $(r=0.87 ; n=29$; $p<0.001$; Fig. 3D) between the number of the Fos-positive neurons in the VLPO cluster at the height of the sleep period and the total number of surviving VLPO neurons (as determined by Nissl staining). Hence, most results were calculated as comparisons of behavior against loss of Fos-IR neurons.

Loss of NREM sleep time and EEG delta power in animals with lesions of the VLPO cluster and extended VLPO

Previous work in our laboratory showed that c-fos expression in the VLPO cluster was positively correlated with sleep during the hour before death (Sherin et al., 1996). We hypothesized that the 

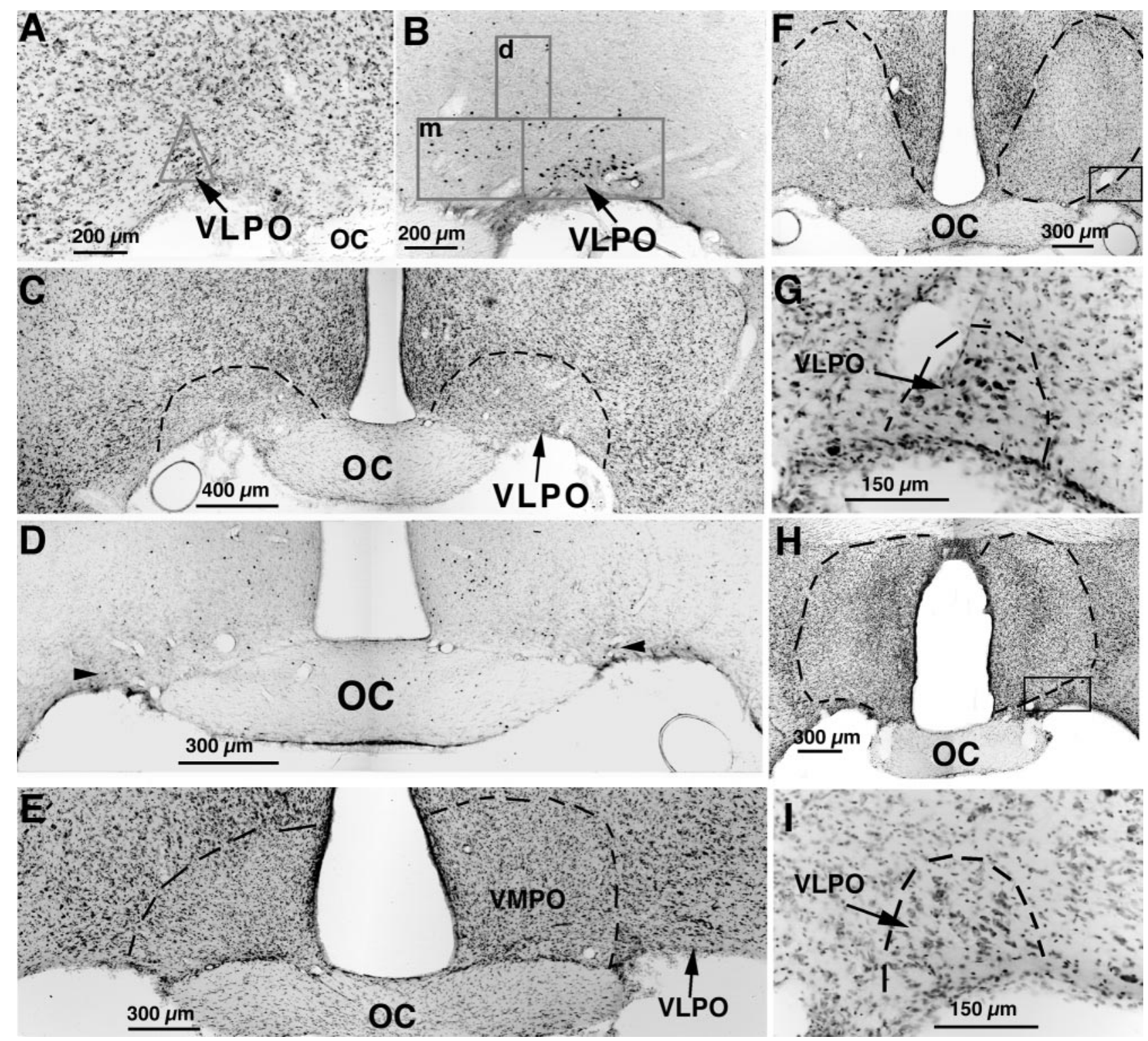

Figure 2. A series of photomicrographs illustrating lesions of the VLPO $(A-D)$ and control lesions $(E-I)$ by ibotenic acid injection. $A$, The VLPO cluster is visualized by Nissl staining as a pyramidal-shaped cell cluster along the base of the brain, just lateral to the optic chiasm. The triangular counting box used to quantify neurons in the VLPO cluster in Nissl preparations is shown. $B$, The VLPO cluster stands out as an aggregation of c-fos-immunoreactive neurons after sleep. The boxes in $B$ represent those used to count cells in the VLPO cluster and the medial $(m)$ - and dorsal $(d)$-extended VLPO. $C, D$, After bilateral ibotenic acid lesions, it is possible to quantify the remaining neurons in the VLPO cluster by Nissl staining ( $C$; dashed lines represent borders of lesions) or by Fos immunostaining ( $D$; the arrowheads indicate Fos-positive cells in the VLPO cluster) in animals that are perfused during the height of the sleep cycle, between 10:00 and 12:00 noon. $E-I$, A lesion of the ventromedial preoptic area (VMPO; E) did little if any damage to the VLPO, and lesions dorsal to the VLPO $(F)$ or larger lesions in the medial preoptic area $(H)$ left the VLPO cluster nearly intact, as shown by higher magnification views of the areas included in the boxes ( $G, I$, respectively).

remaining NREM sleep after VLPO lesions would correlate with the number of surviving neurons in the VLPO cluster that express c-fos at the peak of the sleep cycle. Regression analysis across the entire series of animals with VLPO lesions showed a strong correlation $(r=0.77 ; n=29 ; p<0.001)$ between the reduction of Fos-IR neurons in the VLPO cluster and the percentage of the reduction in NREM sleep per $24 \mathrm{hr}$ (Fig. 3A). Similarly, there was a strong linear relationship between the reduction in the number of Fos-IR neurons in the VLPO cluster and the reduction of delta power in the EEG recording across 24 hr $(r=0.79 ; n=29 ; p<0.001$; Fig. $3 C)$.

The pronounced effect of successful bilateral lesions $(>80 \%$ cell loss) of the VLPO cluster on sleep was apparent in individual rats (Fig. $4 A)$ as well as in grouped data ( $n=7$; Fig. $4 B)$. Reductions were seen in both total sleep time $(56.4 \pm 7.1 \%$, mean \pm SEM; $p<0.01)$ and NREM sleep time $(54.3 \pm 4.3 \% ; p<$ $0.01)$ after VLPO cluster lesions. The duration of individual sleep bouts during $6 \mathrm{hr}$ in the light cycle (7:00-13:00) was reduced from $5.87 \pm 0.66$ to $2.28 \pm 0.19 \min (p<0.01)$, but the number of bouts was actually increased from $38.3 \pm 3.9$ to $51.5 \pm 4.0(p<$ $0.05)$, suggesting that animals with lesions of the VLPO cluster still had strong sleep drive (sleepiness) but could not maintain the sleep state. In addition, there was also a $57.8 \pm 7.4 \%(p<0.002$; $F=11.63)$ reduction of delta power in postlesion compared with 

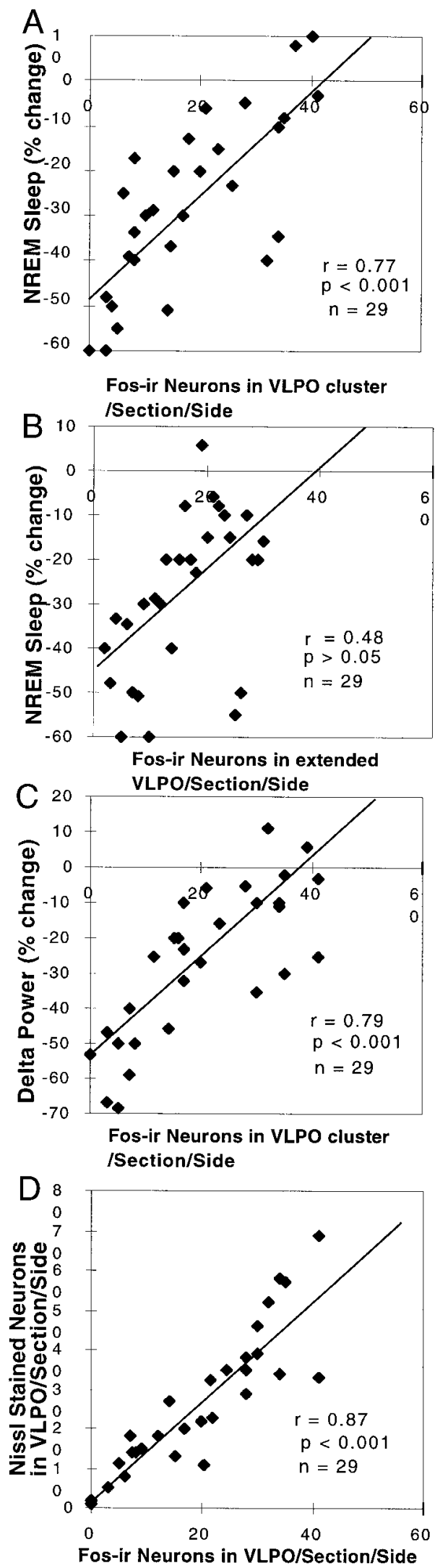

Figure 3. Correlation of the loss of NREM sleep and delta power with the loss of VLPO neurons. $A$, The relationship of the loss of Fos-IR neurons in the VLPO cluster and the percentage reduction of NREM sleep is shown. The NREM sleep reduction was calculated by the percentage change in $24 \mathrm{hr}$ NREM sleep time from before to after the lesion. prelesion measurements in the same group of animals, indicating that the loss of NREM sleep time was not compensated by an increased quality (delta power) of the remaining sleep (Figs. $3 C$, $4 A$ ). It appeared that the reduction in NREM sleep was independent of circadian time (Fig. 4) and that the circadian rhythm of the remaining NREM sleep was not affected by the bilateral VLPO lesions (Fig. 4).

Because all of these lesions also affected the dorsal and medial extensions of the VLPO to varying degrees, the numbers of Fos-IR neurons in the extended VLPO were also compared with the NREM sleep time in the group of animals with extensive lesions of the VLPO cluster (Fig. 3B). There was no significant correlation $(r=0.48 ; p>0.05)$. In a further analysis, we examined the sleep in a group of animals with lesions of the dorsalextended VLPO but not the VLPO cluster $(n=5$; taken from 9 rats in group I with lesions dorsal to the VLPO cluster in which there was $<50 \%$ cell loss in the VLPO cluster). In this group of animals, there was only a $14.6 \pm 9.6 \%$ reduction in NREM sleep time $(p>0.05)$, although delta power across the $24 \mathrm{hr}$ EEG was decreased by almost $28.6 \pm 5.6 \%(p<0.05)$. In a separate group of rats with lesions involving the medial extension of the VLPO but not the VLPO cluster (drawn from the group with ibotenic acid injections aimed at the medial preoptic area; $n=5)$, there was a $24.5 \pm 4.9 \%(p<0.01)$ reduction in NREM sleep time (Fig. $5 A)$ but only an $8.8 \pm 1.3 \%(p>0.05)$ decrease in delta power in the $24 \mathrm{hr}$ EEG. The reduction of NREM sleep time in the rats with medial preoptic lesions occurred mostly during the light period when they typically experience most of their NREM sleep.

\section{Loss of REM sleep with lesions of the VLPO cluster and the} extended VLPO

REM sleep was also decreased by $58.5 \pm 8.8 \%(p<0.05)$ in animals with $>80 \%$ bilateral lesions of the VLPO cluster (Fig. $6 A ; n=7)$. In particular, the REM peaks during the animal's normal waking time (i.e., during the night) were nearly abolished. However, the decrease in REM sleep could not be attributed to injury to the VLPO cluster, because the correlation between the number of Fos-IR neurons in the VLPO cluster and REM sleep time was not statistically significant $(r=0.35$; $p>0.1$; Fig. $6 B)$. However, in all of these cases there was also extensive loss of neurons in the area surrounding the VLPO cluster, including the dorsal- and medial-extended VLPO.

In agreement with these areas being responsible for the loss of REM sleep, rats with lesions solely dorsal to the VLPO cluster, in the dorsal-extended VLPO, showed reductions in REM sleep that averaged twice the reductions in NREM sleep, or $35.4 \pm$ $4.5 \%(n=5 ; p<0.005)$. Rats with bilateral lesions of the medial preoptic area, involving the medial extension of the VLPO but not the VLPO cluster, showed a reduction in REM sleep (30.9 \pm

The number of Fos-IR neurons in the VLPO cluster was measured in rats that were perfused while sleeping between 10:00 and 12:00 noon $(r=$ $0.77 ; p<0.001)$. $B$, The relatively weak correlation $(r=0.48)$ of the loss of Fos-IR neurons in the extended VLPO and the percentage reduction of NREM sleep did not reach statistical significance. $C$, The relationship between the loss of Fos-IR neurons in the VLPO and the percentage reduction of the delta power calculated from comparing the $24 \mathrm{hr}$ prelesion and postlesion EEG $(r=0.79 ; p<0.001)$ is shown. $D$, The number of Nissl-stained neurons in the VLPO cluster correlates closely with the number of Fos-IR neurons in the nucleus during morning sleep $(r=0.87$; $p<0.001)$. 
Figure 4. The effect of severe bilateral $(>80 \%)$ lesions of the VLPO cluster on delta power and NREM sleep. $A$, Comparison of delta power in the EEG plotted over $24 \mathrm{hr}$ for a single animal with $>90 \%$ cell loss in the VLPO cluster bilaterally (R1352) before lesion (gray) and $6 \mathrm{~d}$ after lesion (black) is shown. The black bar along the bottom of the graph indicates the dark cycle. Note the nearly complete loss of periods of greatest delta activity during the first part of the sleep cycle (just after lights on) and during the brief episodes of sleep during the dark cycle. $B$, For the entire group of seven rats with severe bilateral VLPO lesions, there was loss of nearly 55\% of NREM sleep time. In particular, NREM sleep bouts during the dark cycle were almost entirely eliminated. However, circadian variation in NREM sleep time persisted. ${ }^{*} p<0.05$; * $p<0.01$.
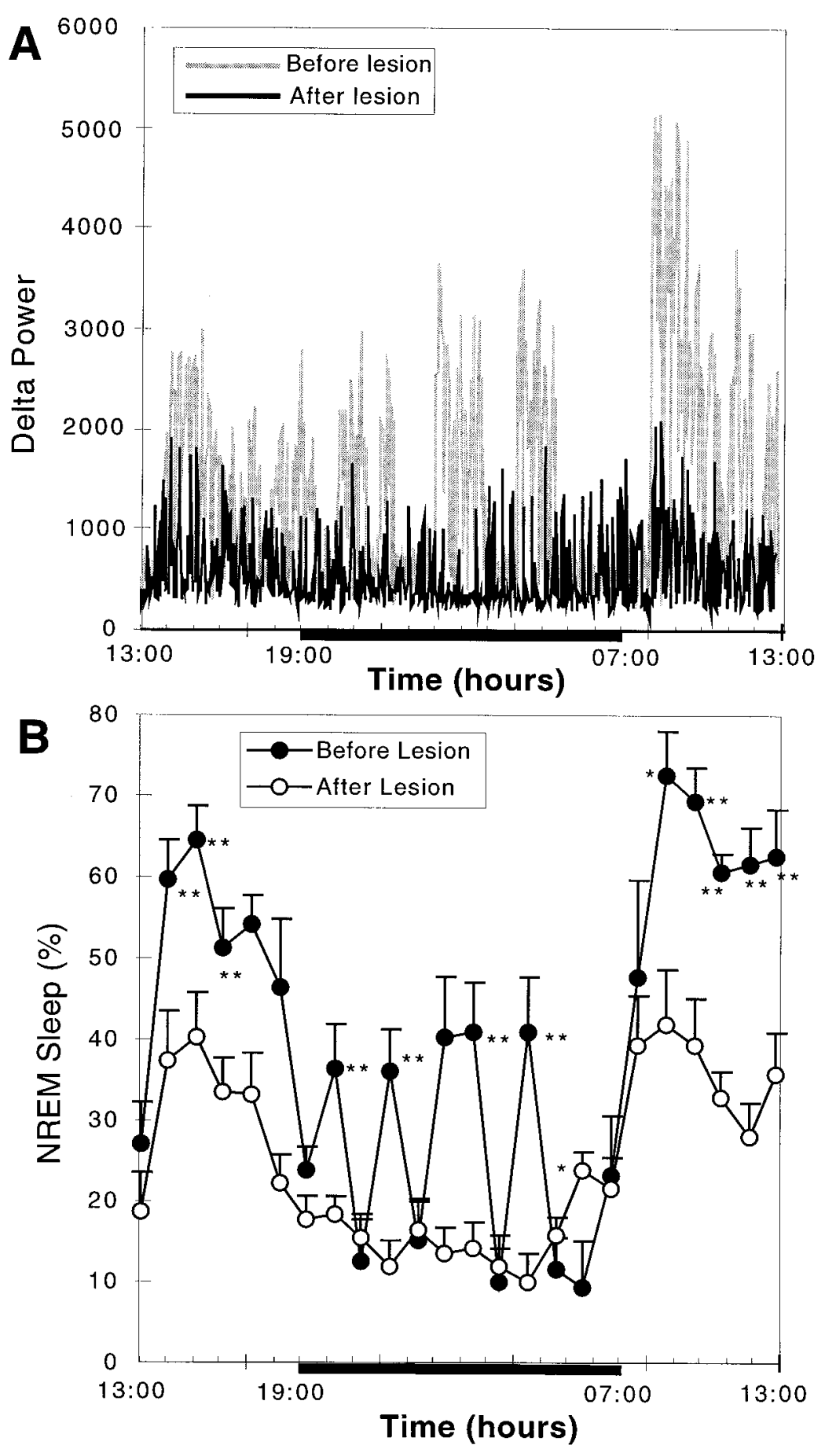

$19.5 \%$ ) similar to the degree of NREM reduction, but this was not statistically significant $(n=5 ; p>0.05)$. It appeared that the reduction in REM sleep time predominantly occurred during the light period (Fig. $5 B$ ). Counts of Fos-IR neurons within the medial- and dorsal-extended VLPO showed a statistically significant correlation $(r=0.74 ; p<0.01 ; n=29)$ with the reduction in REM sleep time (Fig. $6 C$ ).

\section{Experiment 2: long-term effects of bilateral preoptic lesions on sleep and body temperature}

Because the medial preoptic area adjacent to the VLPO plays an important role in thermoregulation (for review, see Satinoff,
1978), we prepared a second group of animals in which we monitored body temperature as well as sleep behavior after preoptic lesions. In addition, we explored the persistence of the changes in sleep and body temperature after preoptic lesions for 3 weeks in a group of 15 additional rats with ibotenic lesions directed at the VLPO, a group of 5 rats with lesions directed at the ventromedial preoptic nucleus, and a group of 6 control rats that received injections of saline into the VLPO region. Within the group with VLPO lesions, Nissl and c-fos staining disclosed that 4 of the 15 rats had $70-90 \%$ bilateral cell loss in the VLPO cluster, 3 had $60-70 \%$ bilateral cell loss in the VLPO cluster, 3 

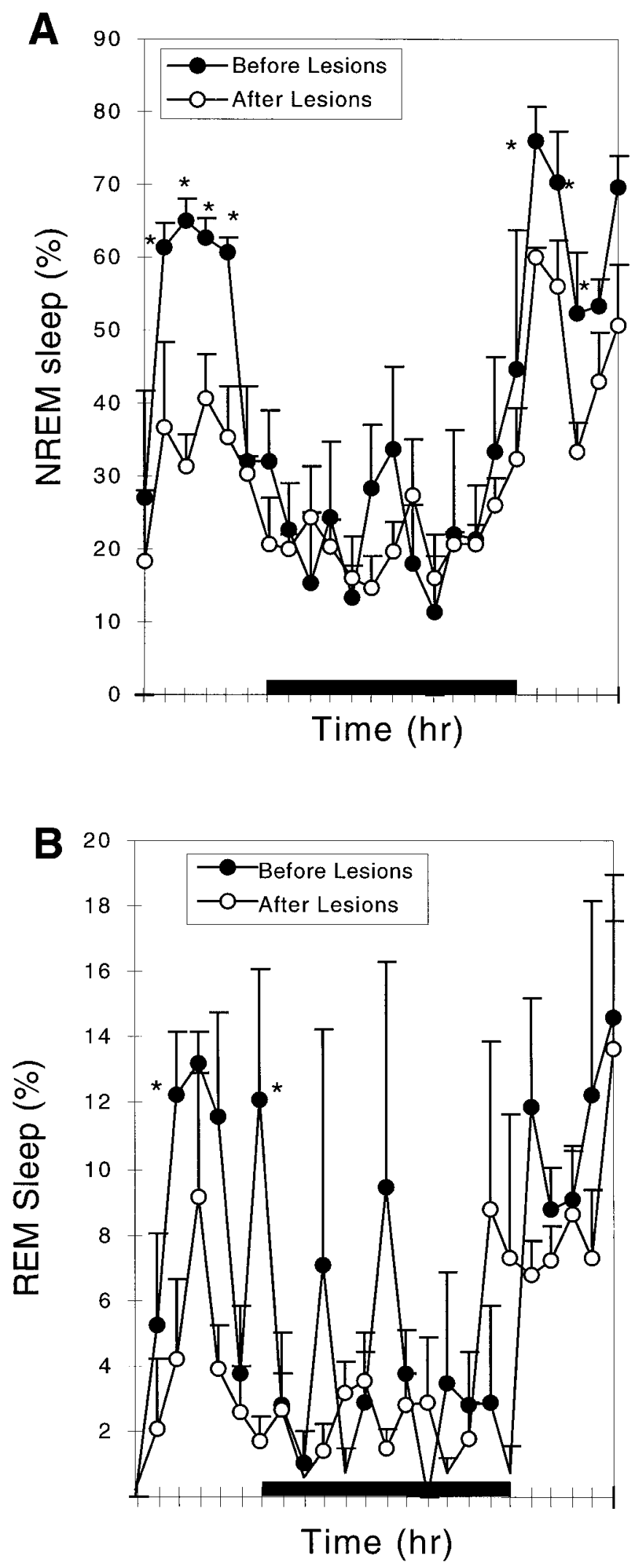

Figure 5. Comparison of NREM and REM sleep before and after lesion of the medial preoptic area. The lesion caused a reduction in NREM sleep by $24.5 \%(A)$ and in REM sleep by $30 \%$ (not statistically significant; $B)$. Loss of NREM sleep was predominantly late in the light cycle, whereas the reduction of REM sleep also involved loss of peaks during the dark cycle. ${ }^{*} p<0.05$. had $70-90 \%$ unilateral cell loss in the VLPO cluster, 2 had lesions ranging from 30 to $60 \%$ of the VLPO cluster neurons, and 3 had no lesion of the VLPO cluster. The small numbers of animals in each group precluded the analysis of damage to the extended VLPO in this series. In the five rats with lesions of the ventromedial preoptic area (Fig. 2E), the lesions were deliberately placed more ventrally and medially than were the medial preoptic lesions in the short-term study to avoid damage to neurons in the medial-extended VLPO (see above).

\section{Effects of lesions of the VLPO cluster}

At the end of 3 weeks after the placement of lesions involving the VLPO cluster, animals appeared healthy and behaved normally. Although specific tests of cognitive or neuroendocrine function were not attempted, the brains showed the same patterns of Fos immunoreactivity as did brains of animals in the acute experiments. NREM sleep was decreased in animals with 70-90\% lesions of the VLPO cluster bilaterally compared with that of saline-injected controls during each of the 3 weeks after lesion placement [first week, reduction by $44.8 \pm 12.5 \%(p<0.05)$; second week, reduction by $62.7 \pm 11.5 \%(p<0.01)$; third week, reduction by $56.7 \pm 12.3 \%(p<0.01)]$. Similarly, REM sleep was reduced by $62.2 \pm 18.3 \%(p<0.01)$ in the first week, $68.7 \pm$ $15.7 \%(p<0.01)$ in the second week, and $74.5 \pm 8.1 \%(p<0.01)$ in the third week. Although, the decrease in NREM sleep actually was more severe in weeks 2 and 3 than in the first week after lesion placement, NREM sleep during weeks 2 and 3 did not differ statistically (Fig. 7A).

There was a strong linear correlation between the NREM sleep reduction across the entire group and the number of Fos-IR neurons in the VLPO cluster $(r=0.75 ; n=21 ; F=22.77 ; p<$ 0.05 ; Fig. $8 A)$ but not in the extended VLPO $(r=0.51 ; p>0.05$; Fig. $8 B$ ). However, the loss of REM sleep correlated with the reduction in the numbers of neurons in the extended VLPO $(r=$ $0.67 ; p<0.05$; Fig. $8 D)$ but not in the VLPO cluster $(r=0.37$; $p>0.05$; Fig. $8 C$ ). In this experiment, EEG/EMG recordings were not monitored before ibotenic acid administration. Therefore we could not analyze delta power (the amplitude of which is highly variable between subjects and hence must be examined in paired prelesion and postlesion data for individual animals).

Although the rats $(n=4)$ with $70-90 \%$ VLPO lesions had a slightly larger amplitude in the range of body temperature $(1.01 \pm$ $0.07^{\circ} \mathrm{C}$; Fig. $\left.9 A\right)$ than did the saline-injected controls $(n=5$; $0.91 \pm 0.07^{\circ} \mathrm{C}$; Fig. $\left.9 B\right)$, the difference was not statistically significant $(p=0.133)$. The mean body temperature showed no significant difference between the two groups (lesion group, $37.3 \pm$ $0.1^{\circ} \mathrm{C}$; control group, $\left.37.63 \pm 0.07^{\circ} \mathrm{C} ; p=0.11\right)$.

\section{Lesions of the ventromedial preoptic nucleus}

By contrast with the larger medial preoptic lesions in the shortterm group, which included the medial-extended VLPO, the ventromedial preoptic lesions caused no change in sleep behavior (total sleep time or circadian rhythm of sleep) (Fig. 7B). However, these animals demonstrated substantial disruption in the ability to regulate body temperature within a narrow range (Fig. $9 C)$. The amplitude of the range of body temperature was $\sim 1.80 \pm 0.20^{\circ} \mathrm{C}$, which was almost double that of control animals $\left(0.91 \pm 0.07^{\circ} \mathrm{C}\right)$. Although the rats with the ventromedial preoptic lesions showed a normal circadian rhythm of body temperature, the mean body temperature was significantly greater than that of controls during the dark period $\left(38.2 \pm 0.1\right.$ vs $37.7 \pm 0.2^{\circ} \mathrm{C} ; p<$ $0.05)$ and significantly lower than that of controls during the light 

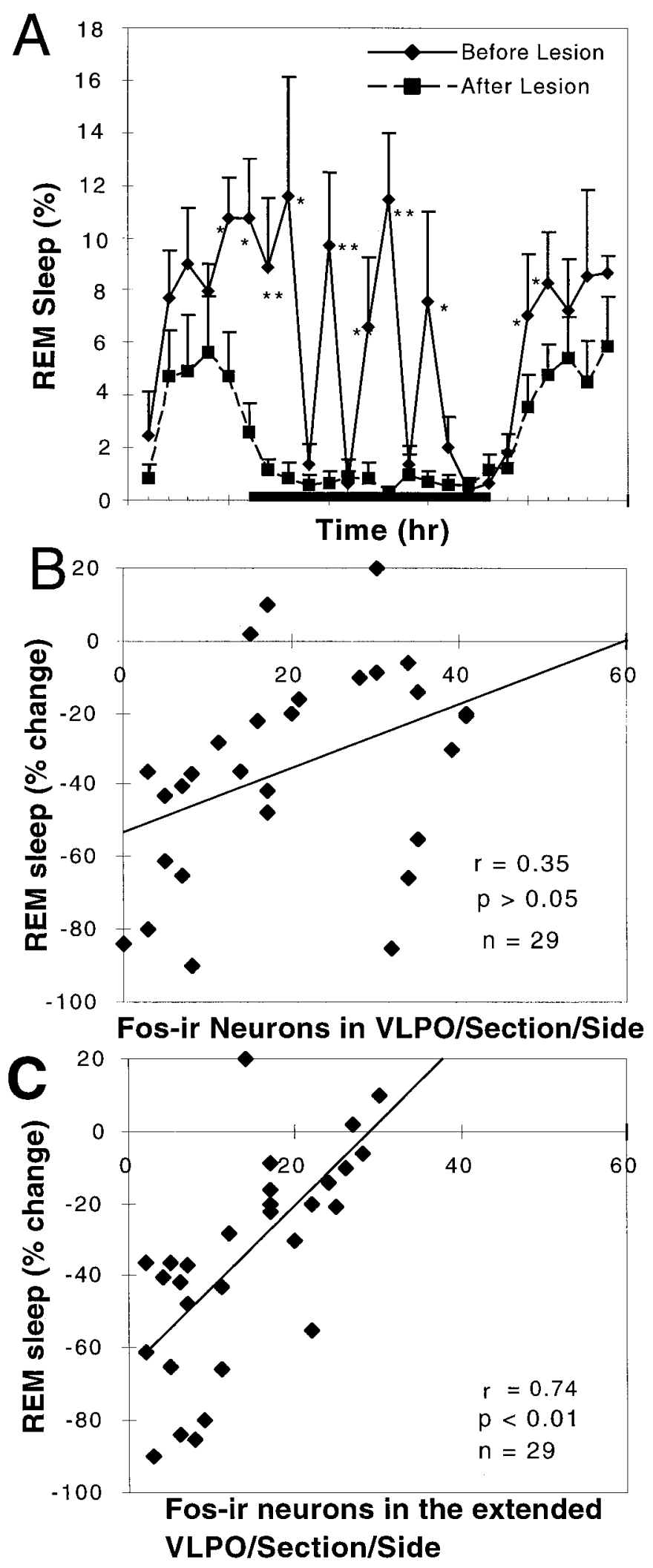

Figure 6. Loss of REM sleep correlates with loss of Fos-IR neurons in the extended VLPO but not in the VLPO cluster. $A$, There was a substantial $(\sim 50 \%)$ loss of REM sleep in the comparison of the prelesion and postlesion behavior of the seven rats with $80-90 \%$ loss of neurons in the VLPO cluster $\left({ }^{*} p<0.05 ;{ }^{*} p<0.01\right)$. REM sleep episodes during the night were almost entirely eliminated. $B$, However, the numbers of surviving Fos-IR neurons in the VLPO cluster after sleep did not corre- period $\left(36.9 \pm 0.2\right.$ vs $\left.37.3 \pm 0.1^{\circ} \mathrm{C} ; p<0.05\right)$, consistent with a wider amplitude of body temperature cycling. In addition, the rats with ventromedial preoptic lesions showed a significant increase in the variance of body temperature compared with that of VLPO-lesioned animals (variance for body temperature in animals with ventromedial preoptic lesions was $6.7 \pm 0.12$; variance for the animals with VLPO lesions was $1.10 \pm 0.05 ; p<0.001$ by $t$ test). We characterize this combination of deficits as a loss of fine tuning of body temperature, similar to the description of Satinoff (1978) in animals with somewhat larger medial preoptic lesions.

\section{DISCUSSION}

Our observations identify a triple dissociation of three different populations of preoptic neurons with principal effects on REM sleep, NREM sleep, and thermoregulation. After bilateral lesions of the VLPO cell cluster, we found a linear correlation between the reduction in the number of sleep-positive cells in the VLPO cluster and reductions in NREM sleep and delta power but not REM sleep. In this group of animals, nearly complete bilateral lesions of the VLPO cell cluster ( $80-90 \%$ cell loss) dramatically reduced NREM sleep by $>50 \%$ and delta power in the $24 \mathrm{hr}$ EEG by nearly $60 \%$. Insomnia caused by lesions of the VLPO cell cluster persisted for at least 3 weeks with no trend toward recovery and was not associated with changes in body temperature. Although the animals with VLPO lesions showed substantial deficits in REM sleep as well, the loss of REM sleep did not correlate with loss of neurons in the VLPO cluster.

By contrast, animals with lesions just dorsal to but not involving the VLPO cell cluster showed statistically significant reductions in REM sleep but not in NREM sleep time. We had argued previously that galanin-immunoreactive neurons in this region that project to the tuberomammillary nucleus represent a part of the VLPO (Sherin et al., 1998). In these experiments, we found that loss of Fos-IR, sleep-positive neurons in the dorsal- and medial-extended VLPO correlated with loss of REM but not NREM sleep, arguing for a specialized role for sleep-positive neurons in the extended VLPO in REM sleep regulation.

Lesions involving the ventromedial preoptic nucleus bilaterally showed dramatic disruption of the fine tuning of thermoregulation, with a larger variance and wider circadian swings in body temperature. However, there were no changes in the periodicity of circadian cycling of body temperature, and the sleep in these animals was normal.

\section{Technical considerations \\ Method for defining lesions}

We used ibotenic acid because it is a cell-specific excitotoxin that does not destroy fibers of passage. We used the smallest practical amount of ibotenic acid (15 nl containing $10 \mathrm{nmol}$ of ibotenic acid), but all of the VLPO lesions included adjacent cell groups to varying degrees, and in no case was the entire VLPO population eliminated. Hence, statistical analysis of the extent of VLPO cell loss compared with physiological results formed a critical component of our analysis.

$\leftarrow$

late well with REM sleep behavior $(r=0.35 ; n=29 ; p>0.05)$. $C$, In contrast, the number of Fos-positive cells in the extended VLPO (in which cell loss was equally substantial in this series) was significantly correlated with the percentage reduction of REM sleep $(r=0.74 ; p<$ $0.01 ; n=29)$. 

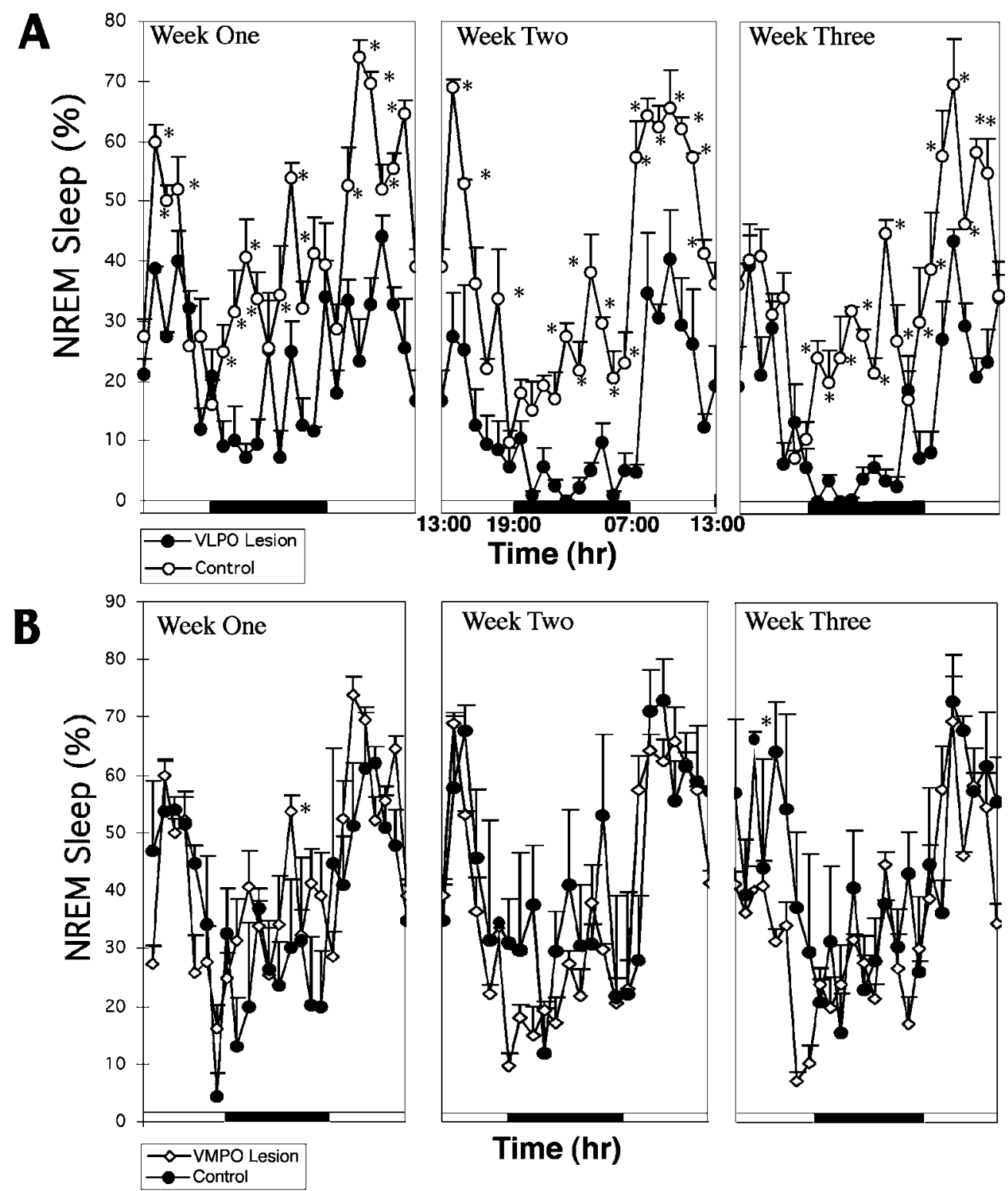

Figure 7. Change in NREM sleep time over a 3 week period after lesions of the VLPO $(A)$ and $V M P O(B) . A$, In four animals with $70-90 \%$ bilateral lesions of the VLPO cluster, the percentage of NREM sleep time was depressed by almost $45 \%$ in the first week, $63 \%$ in the second week, and $57 \%$ in the third week compared with that of a group of control rats that had received bilateral saline injections. As in the short-term experiments, the animals with VLPO lesions showed almost complete loss of NREM sleep during the dark cycle and substantial loss at the onset of the light cycle $\left({ }^{*} p<0.05\right)$. There was no trend toward recovery. $B$, In five animals with severe bilateral lesions of the ventromedial preoptic nucleus, there was no change in NREM sleep time compared with that of saline-injected control animals.

\section{Method for defining the VLPO and the extended VLPO}

The VLPO was originally defined by its unique connections (projections to the histaminergic tuberomammillary nucleus), chemical makeup (cells both galaninergic and GABAergic), and physiological activity (a high percentage of neurons showing Fos expression after sleep) (Sherin et al., 1996, 1998). As is the case for many hypothalamic nuclei, these criteria identify a relatively dense cell cluster as well as a population of adjacent but more scattered neurons with similar chemical, connectional, and physiological characteristics (Sherin et al., 1998; Gaus and Saper, 1999). For the purpose of counting cells in the pyramidal-shaped VLPO cluster in Nissl preparations, it is necessary to use a triangular counting box to avoid counting extraneous neurons. However, in counting Fos-positive VLPO cells, we use a rectangular counting box, because the vast majority of Fos-IR neurons in this region during sleep belong to the VLPO cluster. To capture counts of sleep-positive neurons medial and dorsal to the VLPO cluster, which we consider to be a part of the cell complex, additional counting boxes medial and dorsal to the VLPO were constructed. These counting boxes have the advantage of providing a set of objective criteria for quantification, and to the extent that they do not faithfully follow borders between nuclei, they would be expected to diminish rather than to enhance the positive correlations that we have found.

\section{Interaction of NREM sleep, REM sleep, and thermoregulation in the preoptic region}

Szymusiak et al. (1991) found that the insomnia caused by lesions of the preoptic region in cats can be reversed by placing the animals at a high ambient temperature $\left(33^{\circ} \mathrm{C}\right)$. They hypothesized that sleep is provoked by stimulation of the remaining thermosensitive neurons that stimulate sleep and recently reported that within the VLPO $28 \%$ of sleep-active neurons are warm sensitive as well (Szymusiak et al., 1998). Thus, the sleep deficits reported in previous studies (Nauta, 1946; Sterman and Clemente, 1962a,b) might have in part reflected thermoregulatory deficits.

Our observations indicate that bilateral lesions that destroy 


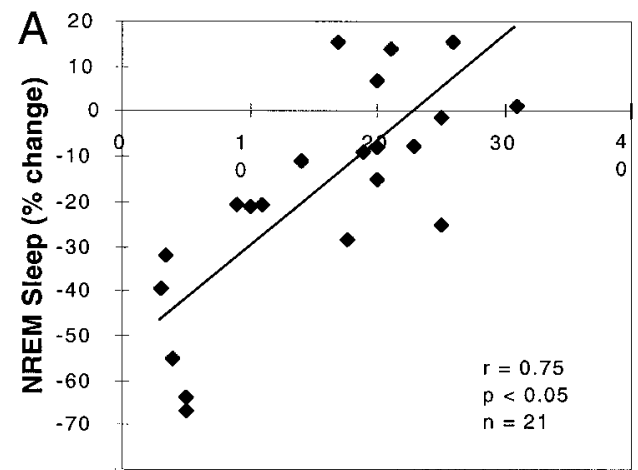

Fos-ir neurons in VLPO cluster/Section/Side

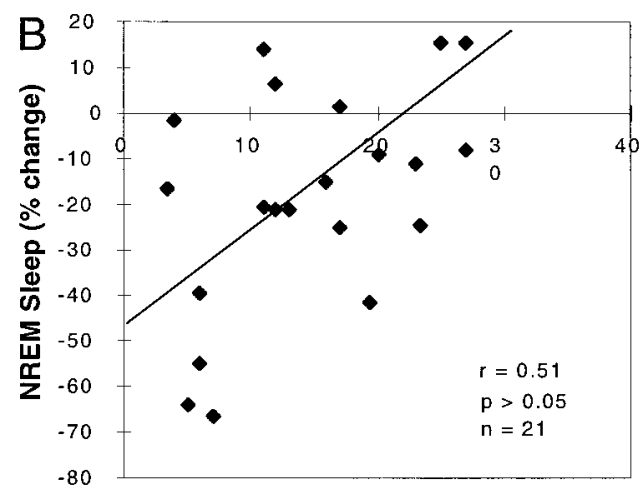

Fos-ir neurons in Extended VLPO/Section/Side

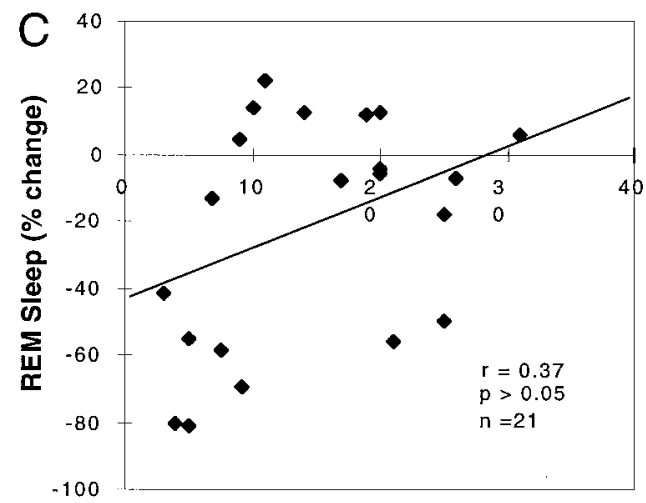

Fos-ir neurons in VLPO cluster/Section/Side

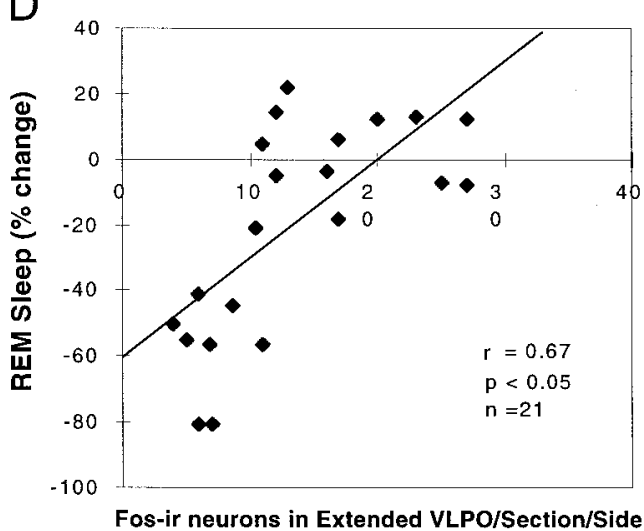

Figure 8. Correlation of loss of NREM or REM sleep time with the numbers of Fos-IR neurons in the VLPO and extended VLPO 3 weeks after placement of severe bilateral lesions (70-90\% cell loss in the VLPO cluster). $A, B$, The decrease in NREM sleep correlated closely with the loss of Fos-IR neurons in the VLPO cluster $(A ; r=0.75 ; p<0.05)$ but not from 80 to $90 \%$ of neurons in the VLPO cell cluster produce profound insomnia but do not alter mean body temperature or its range or circadian pattern over the course of the day. Conversely, lesions in the ventromedial preoptic nucleus, avoiding the VLPO and medial-extended VLPO, caused loss of the fine tuning of body temperature (increased circadian amplitude and variance of body temperature), without affecting circadian periodicity or sleep. Moreover, these alterations persisted for the entire period of 3 weeks that we studied the animals, without any trend toward normalization. Hence, lesions of the preoptic area in previous studies that caused changes in both sleep and body temperature may have involved both the ventromedial preoptic nucleus and the VLPO. For example, Sallanon et al. (1989) found that changes in sleep were not correlated with changes of body temperature, supporting the idea that different populations of the cells in the preoptic area are involved in the regulation of body temperature and sleep. Thus, although neurons involved in sleep and thermal regulation may share considerable input and interact extensively, the sites that ultimately regulate these physiological responses are anatomically and functionally separable.

The dissociation of mechanisms for NREM and REM sleep within the preoptic area is surprising. Previous work, such as that by Benington and Heller (1994), suggested that loss of NREM sleep should result in the proportional loss of REM sleep. Hence, loss of VLPO neurons would be expected to disturb both REM and NREM sleep equally. The lack of correlation of loss of neurons in the VLPO cluster with loss of REM sleep, coupled with the significant correlation of loss of REM sleep with loss of sleep-positive neurons in the medial- and dorsal-extended VLPO, implies that different parts of the VLPO complex may differentially project to different brainstem targets and hence be involved in different phases of sleep.

For example, the region including the medial-extended VLPO was found by Sherin et al. (1998) to project more heavily to the medial part of the tuberomammillary nucleus. In contrast, the dorsal-extended VLPO seems to be responsible for most VLPO projections to the dorsal and medial raphe nuclei (Bjorkum et al., 1999). The role of these different projections in the regulation of sleep remains to be investigated. However, this hypothesis is consistent with the observation that the firing of some medial and dorsolateral preoptic neurons is associated specifically with REM sleep in rats (Koyama and Hayaishi, 1994; Szymusiak et al., 1998).

This view would not preclude interaction by REM and NREM sleep but would suggest that the VLPO is a critical part of the circuitry by which such interaction would take place.

\section{Conclusions}

Although previous studies of the VLPO neurons have correlated their activity with sleep, evidence of a causal role has been lacking. Our results indicate that the VLPO cluster is a necessary component of sleep circuitry, without which NREM sleep is severely impaired. Furthermore, it is likely that in previous studies showing insomnia after large preoptic area lesions, the VLPO was the critical structure responsible for the changes in sleep behavior. To the extent that lesions of the areas medial and dorsal

with the loss of Fos-IR neurons in the extended VLPO $(B ; r=0.51 ; p>$ $0.05)$. $C, D$, On the other hand, the decrease in REM sleep did not correlate with the loss of Fos-IR neurons in the VLPO cluster $(C ; r=$ 0.37; $p>0.05$ ) but did correlate with the loss of Fos-IR neurons in the extended VLPO $(D ; r=0.67 ; p<0.05)$. 


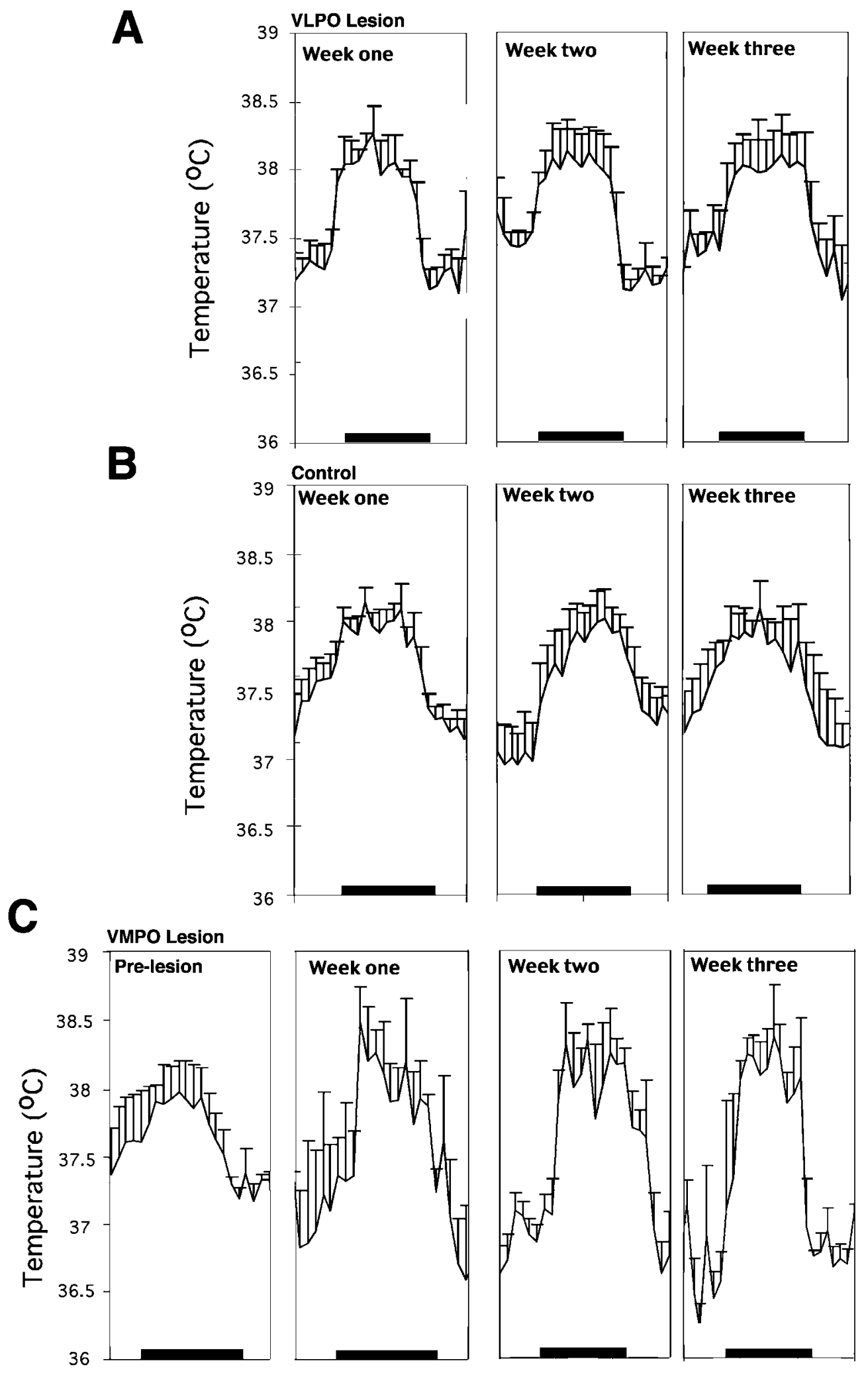

Figure 9. Effect of lesions of the VLPO or ventromedial preoptic nucleus on body temperature recorded over 24 hr at three weekly intervals. $A$, $B$, Severe bilateral lesions of the VLPO in four rats had no effect on the mean body temperature or its daily circadian rhythm $(A)$ when compared with that of four control rats $(B)$. Although the animals with VLPO lesions had a slightly greater circadian excursion of body temperature, this did not differ statistically from that of the saline-injected control animals. $C$, In contrast, in four animals with lesions of the ventromedial preoptic nucleus, the circadian rhythm of body temperature was unaffected, but the range was $\sim 2^{\circ} \mathrm{C}$ during the course of the day (approximately twice normal), and the peaks and troughs of body temperature both differed significantly from that of control animals $(p<0.05)$. Body temperature showed substantial instability in these animals, as evidenced by the significantly larger variance compared with that of the control or VLPO-lesioned animals $(p<0.05)$. 
to the VLPO impair sleep behavior, this is likely to be caused by damage to VLPO neurons that are located outside the main cell cluster, invading these adjacent territories. However, lesions in these regions never produce the profound deficits of NREM sleep and delta power that are associated with lesions of the VLPO cell cluster but rather seem more closely associated with the regulation of REM sleep.

Studies on the long-term effects of sleep deprivation have used models in which sleep deprivation was achieved by continual behavioral stress. The fact that our animals failed to show recovery of sleep behavior even after 3 weeks suggests that VLPO lesions may present a new model for assessing the long-term effects of sleep deprivation on brain function. In addition, study of the mechanisms by which at least a portion of sleep behavior remains in animals with few functional VLPO cells can be used to understand the alternative pathways by which the brain can regulate sleep.

\section{REFERENCES}

Alam MN, McGinty D, Szymusiak R (1995) Neuronal discharge of preoptic/anterior hypothalamic thermosensitive neurons: relation to NREM sleep. Am J Physiol 269:R1240-R1249.

Benington JH, Heller HC (1994) REM-sleep timing is controlled homeostatically by accumulation of REM-sleep propensity in non-REM sleep. Am J Physiol 266:R1992-R2000.

Bjorkum AA, Ha QH, Saper CB (1999) Afferents to and efferents from the raphe nuclei to the ventrolateral preoptic nucleus. Soc Neurosci Abstr 25:625.

Gaus SE, Saper CB (1999) Sleep-active neurons in the ventrolateral preoptic area (VLPO) are galaninergic. Soc Neurosci Abstr 25:625.

John J, Kumar VM (1998) The effects of NMDA lesion of the medial preoptic neurons on sleep and other functions. Sleep 21:587-598.

Koyama Y, Hayaishi O (1994) Firing of neurons in the preoptic/anterior hypothalamic areas in rats: its possible involvement in slow-wave sleep and paradoxical sleep. Brain Res 19:31-38.

Lin JS, Sakai K, Jouvet M (1988) Evidence for histaminergic arousal mechanisms in the hypothalamus of the cat. Neuropharmacology 27:111-122.

Lin JS, Hou Y, Sakai K, Jouvet M (1996) Histaminergic descending inputs to the mesopontine tegmentum and their role in the control of cortical activation and wakefulness in the cat. J Neurosci 16:1523-1537.

McGinty D, Szymusiak R, Thomson D (1994) Preoptic/anterior hypo- thalamic warming increases EEG delta frequency with non-rapid eye movement sleep. Brain Res 667:273-277.

Nauta WJH (1946) Hypothalamic regulation of sleep in rats. An experimental study. J Neurophysiol 9:285-316.

Paxinos G, Watson C (1986) The rat brain in stereotaxic coordinates. San Diego: Academic.

Sallanon M, Denoyer M, Kitahama K, Aubert C, Gay N, Jouvet M (1989) Long-lasting insomnia induced by preoptic neuron lesions and its transient reversal by muscimol injection into the posterior hypothalamus in the cat. Neuroscience 32:669-683.

Satinoff E (1978) Neural organization and evolution of thermal regulation in mammals. Science 201:16-22.

Scammell TE, Griffin JD, Elmquist JK, Saper CB (1998) Microinjection of a cyclooxygenase inhibitor into the anteroventral preoptic region attenuates LPS fever. Am J Physiol 274:R783-R789.

Sherin JE, Shiromani PJ, McCarley RW, Saper CB (1996) Activation of ventrolateral preoptic neurons during sleep. Science 271:216-219.

Sherin JE, Elmquist JK, Torrealba F, Saper CB (1998) Innervation of histaminergic tuberomammillary neurons by GABAergic and galaninergic neurons in the ventrolateral preoptic nucleus of the rat. J Neurosci 18:4705-4721.

Steininger TL, Stevens DR, Hass HL, McGinty D, Szymusiak D (1997) Preoptic area inhibition of histaminergic tuberomammillary neurons in vitro. Assoc Prof Sleep Soc Abstr 2.

Steininger TL, Alam MN, Gong H, Szymusiak R, McGinty D (1999) Sleep-waking discharge of neurons in the posterior lateral hypothalamus of the albino rat. Brain Res 840:138-147.

Sterman MB, Clemente CD (1962a) Forebrain inhibitory mechanisms: cortical synchronization induced by basal forebrain stimulation. Exp Neurol 6:91-102.

Sterman MB, Clemente CD (1962b) Forebrain inhibitory mechanisms: sleep patterns induced by basal forebrain stimulation. Exp Neurol 6:103-117.

Szymusiak R, McGinty D (1986) Sleep suppression following kainic acid-induced lesions of the basal forebrain. Exp Neurol 566:598-614.

Szymusiak R, Danowski J, McGinty D (1991) Exposure to heat restores sleep in cats with preoptic/anterior hypothalamic cell loss. Brain Res 541:134-138.

Szymusiak R, Alam N, Steininger TL, McGinty D (1998) Sleep-waking discharge patterns of ventrolateral preoptic/anterior hypothalamic neurons in rats. Brain Res 803:178-188.

von Economo C (1930) Sleep as a problem of localization. J Nerv Ment Dis 71:249-259.

Yang QZ, Hatton GI (1997) Excitatory and inhibitory inputs to histaminergic tuberomammillary nucleus. Brain Res 773:162-172. 